1 Tumor suppressor BTG1 limits activation of BCL6 expression downstream of ETV6-

2 RUNX1

3 Esther Tijchon ${ }^{1}$, Liesbeth van Emst ${ }^{1}$, Laurensia Yuniati ${ }^{1}$, Dorette van Ingen Schenau ${ }^{1}$,

4 Mylène Gerritsen ${ }^{1}$, Laurens $T$ van der Meer ${ }^{1}$, Owen Williams ${ }^{2}$, Peter M. Hoogerbrugge ${ }^{3}$,

5 Blanca Scheijen ${ }^{1,4^{*}}$ and Frank N. van Leeuwen ${ }^{1 *}$

$6{ }^{1}$ Laboratory of Pediatric Oncology, Radboud university medical center, Nijmegen, the

$7 \quad$ Netherlands

$8{ }^{2}$ Molecular Haematology and Cancer Biology Unit, UCL-Institute of Child Health, London,

$9 \quad$ United Kingdom

${ }^{3}$ Princess Máxima Center for Pediatric Oncology, Utrecht, the Netherlands

${ }^{4}$ Department of Pathology, Radboud university medical center, Nijmegen, the Netherlands

12 * Contributed equally to this work as joined senior authors.

Correspondence to Frank N. van Leeuwen, Laboratory of Pediatric Oncology, Radboud

University Medical Center. Geert Grooteplein Zuid 10, 6525 GA Nijmegen, the Netherlands.

Phone: $\quad+31$ 24

3666203.

Fax:+31

24

3666352.

E-mail:

\title{
17 FrankN.vanLeeuwen@radboudumc.nl
}

Category: Malignant hematopoiesis

Word count: 1498 
Highlights

- Loss of BTG1 potentiates ETV6-RUNX1-mediated self-renewal capacity

- $\quad$ Tumor suppressor BTG1 limits activation of BCL6 by ETV6-RUNX1

- BCL6 enhances the clonogenicity of primitive B lymphoid progenitors

\section{Abstract}

Translocation $t(12 ; 21)$ (p13;q22), giving rise to the ETV6-RUNX1 fusion gene, is the most common genetic abnormality in childhood B-cell precursor acute lymphoblastic leukemia (BCP-ALL). This translocation usually arises in utero, but its expression is insufficient to induce leukemia and requires other cooperating genetic lesions for BCP-ALL to develop. Deletions affecting the transcriptional coregulator BTG1 are frequently observed in ETV6-RUNX1-positive leukemia. Here we show that Btg1 deficiency enhances the self-renewal capacity of ETV6-RUNX1 positive mouse fetal liver derived hematopoietic progenitors (FL-HPCs). Combined expression of the fusion protein and a loss of BTG1 drives upregulation of the proto-oncogene $B c / 6$ and downregulation of BCL6 target genes, such as p19Arf and Tp53. Similarly, ectopic expression of BCL6 promotes the self-renewal and clonogenic replating capacity of FL-HPCs, by suppressing the expression of p19Arf and Tp53.

Together these results identify BCL6 as a potential driver of ETV6-RUNX1 mediated leukemogenesis, which could involve loss of BTG1 dependent suppression of ETV6RUNX1 function.

\section{Introduction}

B-cell precursor acute lymphoblastic leukemia (BCP-ALL) is the most common malignancy in children and characterized by the presence of distinct genetic abnormalities. The $t(12 ; 21)$ (p13;q22) chromosomal translocation arises in utero, giving rise to ETV6-RUNX1 fusion protein, generating a preleukemic clone, which requires additional cooperating events before 
overt leukemia develops [1, 2]. Genomic profiling of ETV6-RUNX1-positive BCP-ALL has identified recurrent copy number aberrations (CNAs), including deletions affecting $B$-cell translocation gene 1 (BTG1) [3, 4]. We and others have shown that monoallelic BTG1 deletions in ETV6-RUNX1-positive BCP-ALL are the result of genomic rearrangements mediated by aberrant RAG recombinase activity $[5,6]$. Focal deletions affecting BTG1 are present in $9 \%$ of all BCP-ALL cases, but are enriched to about $20 \%$ in ETV6-RUNX1-positive leukemia, without affecting patient outcome $[3,4,6,7]$. BTG1 functions as a transcriptional cofactor that acts by recruiting effector molecules, such as the protein arginine methyltransferase I (PRMT1) to specific transcription factors, thus affecting proliferation and differentiation $[8,9]$. In this paper, we investigated if loss of BTG1 affects the self-renewal capacity of ETV6-RUNX1 in mouse fetal liver hematopoietic progenitor cells (FL-HPCs) by performing clonogenic B cell progenitor assays and gene expression analysis.

\section{Methods}

\section{Mice}

The generation of C57BL/6J Btg1 knockout (KO) mice has been previously described by Farioli-Vecchiolo et al. [10]. The animals were maintained under specific pathogen-free conditions at our animal facility. All animal experiments were approved by the Animal Experimental Committee of the Radboud university medical center and were performed in accordance with institutional and national guidelines. For the retroviral transduction experiments, fetal liver cells derived from day 13.5 of wild-type and $B \operatorname{tg} 1^{-/}$embryos were used.

\section{Retroviral transduction of fetal liver-derived progenitor cells and adult bone marrow}

Primitive fetal liver hematopoietic progenitor cells (FL-HPCs) were purified as c-Kit ${ }^{+}$Ter119 cells from fetal livers of embryonic day 13.5 (E13.5) of C57BI6/J wild-type and Btg $1^{-/}$mice by MACS sort using Ter119 and cKit (CD117) beads (Miltenyi Biotec). FL-HPCs were expanded 
in Iscoves modified Dulbecco medium (IMDM) (Gibco) supplemented with $10 \%$ fetal-bovine serum (FBS) (Greiner Bio-One), 1\% Penicillin-Streptomycin (Invitrogen) and 55 $\mu \mathrm{M}$ 2-ME (Gibco) in the presence of $10 \mathrm{ng} / \mathrm{ml}$ fms-like tyrosine kinase-3 ligand (Flt-3L), 20ng/ml Interleukin 7 (IL-7) ( and 100ng/ml stem cell factor (SCF) (all R\&D) for 24 hours. Cells were transduced with retrovirus by spinoculation for $45 \mathrm{~min}$ at $700 \mathrm{xg}\left(25^{\circ} \mathrm{C}\right)$ in the presence of $5 \mu \mathrm{g} / \mathrm{ml}$ polybrene and cultured with the same growth factors for 48 hours, as has been described by Morrow et al. [11]. CD19+ bone marrow cells were purified from 10 weeks old wild-type and $B \operatorname{tg} 1^{-/}$mice by MACS sorting using CD19 beads (Miltenyi Biotec). CD19+ cells were cultured in improved minimal essential medium (IMEM) (Gibco) supplemented with $2 \%$ FBS (Greiner Bio-One), 1\% Penicillin-Streptomycin (Invitrogen), 0.03\% primatone and 55 $\mathrm{M}$ 2-ME in the presence of $20 \mathrm{ng} / \mathrm{ml} \mathrm{IL-7} \mathrm{(R \& D)} \mathrm{on} \mathrm{OP9} \mathrm{stroma} \mathrm{for} 48$ hours. Cells were transduced by spinoculation $\left(45 \mathrm{~min}\right.$ at $700 \mathrm{xg}, 25^{\circ} \mathrm{C}$ ) in the presence of $10 \mu \mathrm{g} / \mathrm{ml}$ protamine sulphate and cultured in the same pro-B cell medium for 24 hours. Transduction efficiency was determined by analyzing EGFP expression using flow cytometry.

\section{B-cell colony-forming assays}

Transduced FL-HPCs $\left(1 \times 10^{4}\right.$ cells/plate) and CD19+ bone marrow cells $\left(1 \times 10^{5}\right.$ cells/plate) derived of wild-type and $B \operatorname{tg} 1^{--}$animals, were cultured in duplicate methylcellulose cultures using 35mm plates (M3630, STEMCELL Technologies), 48 hours after viral transduction. The FL-HPC methylcellulose cultures were supplemented with 10ng/ml FLT3L, 20ng/ml IL-7 and $100 \mathrm{ng} / \mathrm{ml}$ SCF . After 6-10 days, colonies (>30 cells) were scored, pooled and counted. Replating of $1 \times 10^{4}$ cells/plate was performed under identical conditions.

\section{Results and discussion}

To examine contribution of BTG1 loss to ETV6-RUNX1 positive ALL, we compared in vitro self-renewal capacity of wild-type and Btg1-deficient FL-HPCs. Therefore, FL-HPCs isolated from C57BI/6J wild-type and Btg $1^{-/}$embryos at 13.5 days post coitum (dpc) were transduced 
with either control or ETV6-RUNX1 retrovirus and propagated in B-lineage promoting culture conditions. Transduction was observed in $40 \%-70 \%$ of both wild-type and $B \operatorname{tg} 1^{-/}$FL-HPCs by flow cytometry and confirmed by western blot analysis (Supplementary Figure E1). Wild-type and $\mathrm{Btg}^{-/} \mathrm{FL}-\mathrm{HPCs}$ transduced with either control or ETV6-RUNX1 virus were cultured in methylcellulose containing cytokines to facilitate expansion and differentiation of early B cell progenitors. Subsequently, cells were analyzed for their ability to maintain self-renewal capacity by serial replating assays. We observed that introduction of the ETV6-RUNX1 fusion protein enhanced self-renewal capacity, particularly in a Btg1-deficient background (Fig. 1A). However, transformation to a full leukemia in vivo by transplantation of ETV6RUNX1 transduced wild-type and Btg $1^{-/}$HPCs was not observed (results not shown). These data suggest that BTG1 function limits the ETV6-RUNX1-mediated self-renewal capacity of colony-forming fetal progenitor B-cells, but that loss of Btg1 is not sufficient to drive leukemogenesis in this experimental model.

To determine whether this phenotype could also be observed in adult-type B cell progenitors, we examined the effect of ETV6-RUNX1 expression in CD19+-purified adult bone marrow cells, derived from wild-type or $\mathrm{Btg}^{-1}$ mice. Consistent with previous reports [12], ETV6-RUNX1 suppressed colony formation of adult progenitor B-cells, which was further enhanced in the absence of BTG1 (Supplementary Figure E2). These findings are consistent with the notion that ETV6-RUNX1-mediated leukemic transformation occurs primarily in the developing embryo and not in adult progenitor B-cells.

To elucidate the molecular mechanism by which Btg1 deficiency enhances ETV6RUNX1-induced self-renewal capacity of FL-derived progenitor B-cells, we performed gene expression analysis of FL-HPCs cultured in our standard cytokine-containing medium. Gene Set Enrichment Analysis (GSEA) was performed to define KEGG pathways that were specifically affected by ETV6-RUNX1 expression in the two different genetic backgrounds (Fig. 1B and Supplementary Table E1). Interestingly, we identified a p53 signature in both wild-type and $\mathrm{Btg}^{-1}$ FL-HPCs in the presence of ETV6-RUNX1 expression, which involved downregulation of p53 target genes Cdkn2a, Gadd45g and CcnD2 expression 
133 (Supplementary Table E1). As we observed reduced expression of Tp53 in ETV6RUNX1;Btg $1^{-1}$ fetal progenitor B-cells, we hypothesized that an upstream regulator of the p53 pathway might be affected in these cells. Notably, we observed a 4-fold upregulation of Bcl6 mRNA levels in ETV6-RUNX1-positive Btg1-deficient fetal progenitor B-cells relative to control cells. By qRT-PCR we validated that Bcl6 expression was induced in ETV6RUNX1;Btg $1^{-/}$pro-B cells, accompanied by repression of known BCL6 target genes such as p19Arf, Tp53, CcnD2, Bcl2 and c-Myc (Fig. 1C). However, due to poor quality of the available antibodies, we were unable to detect native BCL6 protein in these fetal liver cells.

As p53 is subject to regulation of the E3 ubiquitin-protein ligase MDM2, and ETV6RUNX1 has been shown to activate MDM2 expression [13], we examined Mdm2 mRNA expression in these FL-HPCs. However, no significant differences in expression of Mdm2 were found (Fig. 1C). By western blot analysis we confirmed that p53 protein levels were reduced in ETV6-RUNX1-positive fetal progenitor B-cells relative to control and $B \operatorname{tg} 1^{-/-}$cells, while expression was even further reduced in ETV6-RUNX1;Btg1 $1^{-}$pro-B-cells (Fig. 1D). These data demonstrate that BTG1 loss synergizes with ETV6-RUNX1 in activating BCL6 expression and repression of its downstream targets such as Tp53 and p19Arf.

To study whether increased BCL6 expression itself was responsible for the enhanced self-renewal capacity observed in ETV6-RUNX1-positive Btg1 ${ }^{-1}$ FL-HPCs, we transduced wild-type FL-derived progenitor B-cells with either control or BCL6 retrovirus. Flow cytometric analysis showed a transduction efficiency of 30\%-50\% GFP-positive cells (data not shown). Indeed, forced expression of BCL6 efficiently promoted the clonogenic replating capacity of FL-HPCs to a similar extent as observed in ETV6-RUNX1;Btg1\% cells (Fig. 2A). Gene expression analyses by qRT-PCR revealed a significant reduction in p19Arf and Tp53 expression, which mirrored the phenotype observed in ETV6-RUNX1;Btg $1^{-1}$ fetal progenitor B-cells (Fig. 2B). Western blot analysis confirmed that p53 protein levels were decreased in BCL6 transduced fetal pro-B-cells (Fig. 2C). These results indicate that BCL6 enhances the clonogenicity of primitive B lymphoid progenitors, which is in sharp contrast to its effects on adult definitive precursor B-cells [14]. 
Previous studies have shown that RUNX1 is methylated by the arginine methyltransferase PRMT1 [15-17] and that BTG1 acts by recruiting and stimulating the enzymatic activity of PRMT1 $[8,9,18]$. Consistent with these findings, we could demonstrate that ETV6-RUNX1 associates with both BTG1 and PRMT1 when co-expressed in HEK293 cells (Fig. 3A-B). In addition, by performing PRMT1 co-immunoprecipitation in control and $B \operatorname{tg} 1^{1 / 2}$ FL-HPCs protein lysates, we observed that BTG1 is required for the association between ETV6-RUNX1 and PRMT1 (Fig. 3C). However, how modulation of ETV6-RUNX1 function by the BTG1-PRMT1 complex affects expression of BCL6 remains to be explored.

BCL6 has been identified as a critical determinant of leukemic stem cell survival in chronic myeloid leukemia $(C M L)$ and $B C R-A B L 1$-positive $A L L$, involving repression of p14ARF and TP53 $[14,19]$. Previous reports have shown that the RUNX1 fusion protein AML1-ETO, an important driver of acute myeloid leukemia, represses transcription of p14ARF [20]. Our study shows that downstream targets of BCL6, including p19Arf and Tp53, are downregulated in ETV6-RUNX1-positive fetal progenitor B-cells, particularly in the absence of BTG1 function. Furthermore, ectopic expression of BCL6 in primitive progenitor B-cells induced a similar proliferative expansion, accompanied by downregulation of TP53 and p19Arf expression. These data indicate that BCL6 promotes proliferation of primitive B lymphoid progenitors by suppressing the p53 pathway. Future studies will need to establish whether inhibition of BCL6 could be effective in the eradication of leukemia-initiating cells in ETV6-RUNX1-positive ALL.

Acknowledgements: The authors thank R. Bernards for -BCL6 retroviral constructs. This research was supported by a grant from the Stichting Kinderen Kankervrij (KiKa-77).

Potential conflict of interest: The authors declare no competing financial interests. 
1. Ford AM, Bennett CA, Price CM, Bruin MC, Van Wering ER, Greaves M. Fetal

2. Greaves MF, Wiemels J. Origins of chromosome translocations in childhood leukaemia. Nat Rev Cancer. 2003;3:639-649.

3. Kuiper RP, Schoenmakers EF, van Reijmersdal SV, et al. High-resolution genomic profiling of childhood ALL reveals novel recurrent genetic lesions affecting pathways involved in lymphocyte differentiation and cell cycle progression. Leukemia. 2007;21:1258-1266.

4. Mullighan CG, Goorha S, Radtke I, et al. Genome-wide analysis of genetic alterations in acute lymphoblastic leukaemia. Nature. 2007;446:758-764.

5. Papaemmanuil E, Rapado I, Li Y, et al. RAG-mediated recombination is the predominant driver of oncogenic rearrangement in ETV6-RUNX1 acute lymphoblastic leukemia. Nat Genet. 2014;46:116-125.

6. Waanders E, Scheijen B, van der Meer LT, et al. The origin and nature of tightly clustered BTG1 deletions in precursor B-cell acute lymphoblastic leukemia support a model of multiclonal evolution. PLoS Genet. 2012;8:e1002533.

7. Matsuda $S$, Rouault J, Magaud $J$, Berthet $C$. In search of a function for the TIS21/PC3/BTG1/TOB family. FEBS letters. 2001;497:67-72.

8. Berthet C, Guehenneux F, Revol V, et al. Interaction of PRMT1 with BTG/TOB proteins in cell signalling: molecular analysis and functional aspects. Genes Cells. 2002;7:29-39.

9. Lin WJ, Gary JD, Yang MC, Clarke S, Herschman HR. The mammalian immediateearly TIS21 protein and the leukemia-associated BTG1 protein interact with a protein-arginine N-methyltransferase. J Biol Chem. 1996;271:15034-15044.

10. Farioli-Vecchioli S, Micheli L, Saraulli D, et al. Btg1 is Required to Maintain the Pool of Stem and Progenitor Cells of the Dentate Gyrus and Subventricular Zone. Front Neurosci. 2012;6:124.

11. Morrow M, Horton S, Kioussis D, Brady HJ, Williams O. TEL-AML1 promotes development of specific hematopoietic lineages consistent with preleukemic activity. Blood. 2004;103:3890-3896.

12. Fischer $M$, Schwieger $M$, Horn $S$, et al. Defining the oncogenic function of the TEL/AML1 (ETV6/RUNX1) fusion protein in a mouse model. Oncogene. 2005;24:7579-7591.

13. Kaindl $U$, Morak M, Portsmouth $C$, et al. Blocking ETV6/RUNX1-induced MDM2 overexpression by Nutlin-3 reactivates p53 signaling in childhood leukemia. Leukemia. 2014;28:600-608.

14. Hurtz C, Hatzi K, Cerchietti L, et al. BCL6-mediated repression of p53 is critical for leukemia stem cell survival in chronic myeloid leukemia. $J$ Exp Med. 2011;208:2163-2174.

15. Herglotz J, Kuvardina ON, Kolodziej S, et al. Histone arginine methylation keeps RUNX1 target genes in an intermediate state. Oncogene. 2013;32:2565-2575.

16. Vu LP, Perna F, Wang L, et al. PRMT4 blocks myeloid differentiation by assembling a methyl-RUNX1-dependent repressor complex. Cell Rep. 2013;5:1625-1638.

17. Zhao X, Jankovic V, Gural A, et al. Methylation of RUNX1 by PRMT1 abrogates SIN3A binding and potentiates its transcriptional activity. Genes Dev. 2008;22:640-653. 
18. van Galen JC, Kuiper RP, van Emst L, et al. BTG1 regulates glucocorticoid receptor autoinduction in acute lymphoblastic leukemia. Blood. 2010;115:48104819.

Figures

Figure 1. BTG1 loss enhances self-renewal of ETV6-RUNX1-positive fetal progenitor B-cells by activation of BCL6 pathway. (A-D) Fetal liver-derived hematopoietic progenitor cells (FLHPCs) were isolated from wild-type (WT) and Btg $1^{-/}$embryos at day $13.5 \mathrm{dpc}$ and transduced with control and ETV6-RUNX1 retrovirus. (A) Control and ETV6-RUNX1 transduced FLHPCs $\left(1 \times 10^{4}\right.$ cells) were seeded 48 hours after transduction in duplicate in B cell specific methylcellulose supplemented with FLT3L, IL-7 and SCF. Serial replating was performed under identical conditions. Mean colony counts (and SEM) were determined (>30 cells/colony) after 6-10 days of culture. Data set is representative of 2 independent experiments. ${ }^{*}, P<0.05,{ }^{* *}, P<0.01$. (B)

(B) Gene set enrichment analyses on gene expression data of WT, ETV6-RUNX1, Btg1 $1^{-\kappa}$ and ETV6-RUNX1;Btg $1^{-}$fetal progenitor B-cells. Total RNA was extracted from FL-HPCs, which were expanded for 12 days in liquid medium supplemented with FLT-3L, IL-7 and SCF. The data were RMA normalized and relative gene expression differences of $>1.6$-fold were included. KEGG pathway analysis was performed using the online Gene Set Enrichment Analysis (GSEA) tool and relevant pathways that are differentially expressed between wild-type and ETV6-RUNX1 as well ETV6-RUNX1;Btg $1^{-1-}$ FL cells are indicated. (C) Relative expression levels of Bcl6, Tp53, p19Arf, Mdm2, CcnD1, CcnD2, Bcl2 and $c-M y c$ were determined by quantitative reverse-transcription PCR (qRTPCR) and normalized to the expression of the housekeeping gene TATA box binding protein (TBP). Data represent the mean and SEM of three independent experiments. ${ }^{*}, P<0.05,{ }^{* *}$, 
expanded for 12 days in liquid medium supplemented with FLT-3L, IL-7 and SCF. The immunoblot demonstrates expression of $\mathrm{p} 53$, while $\beta$-actin serves as a loading control. Quantitation of relative expression levels is indicated.

Figure 2. BCL6 stimulates self-renewal of early B cell progenitors. (A-C) Fetal liver-derived hematopoietic progenitor cells (FL-HPCs) were isolated from wild-type embryos at day 13.5dpc and transduced with control or BCL6 retrovirus. (A) Control and BCL6 transduced FL-HPCs $\left(1 \times 10^{4}\right.$ cells) were cultured 48 hours after transduction in B cell specific methylcellulose supplemented with FLT-3L, IL-7 and SCF. Serial replating was performed under identical conditions. Mean colony counts (and SEM) were determined (>30 cells/colony) after 6-10 days of culture. Data set is representative of 2 independent experiments. ${ }^{* *}, P<0.01$. (B) RNA was isolated from FL-HPCs that were expanded for 10 days in liquid cultures in the presence of cytokines FLT-3L, IL-7 and SCF. Relative expression levels of Tp53, p19Arf, Mdm2, CcnD1, CcnD2, Bcl2 and c-Myc were determined on cDNA generated from transduced wild-type FL progenitor cells by real-time PCR and normalized to TBP. Data represent the mean and SEM of three independent experiments. *, $P<0.05,{ }^{* *}, P<0.01,{ }^{* *}, P<0.001$. (C) Protein lysates were generated from FL-HPCs that were expanded for 13 days in liquid cultures in the presence of cytokines FLT-3L, IL-7 and SCF. The immunoblot shows expression of p53 and the loading control $\beta$-actin. Quantitation of relative expression levels is indicated.

Figure 3. ETV6-RUNX1 interacts with PRMT1 and its cofactor BTG1. (A) HEK293 cells were transfected with expression plasmids encoding HA-ETV6-RUNX1 and FLAG-BTG1 and treated for 16 hours with $5 \mu \mathrm{M}$ of the proteasome inhibitor MG132. Protein lysates were generated and subjected to immunoprecipitation (IP) by incubation with FLAG antibody (Ab). Immunoblot demonstrates the expression of BTG1 using a FLAG-Ab and ETV6-RUNX1 with a HA-Ab in whole cell extracts (WCE) and IP samples. (B) HEK293 cells were transfected 
299 with expression plasmids encoding HA-ETV6-RUNX1 and PRMT1 and treated for 16 hours 300 with $5 \mu \mathrm{M}$ of the proteasome inhibitor MG132. Protein lysates were subjected to IP using HA301 Ab. The immunblot shows expression of PRMT1 using a PRMT1-Ab and ETV6-RUNX1 with 302 a HA-Ab in WCE and IP. (C) FL-HPCs (cKit ${ }^{+}$Ter119-) were isolated from wild-type and Btg $1^{-/}$ 303 embryos at day 13.5dpc and transduced with control and ETV6-RUNX1 virus. Protein lysates 304 were generated from FL-HPCs that were expanded for 12 days in liquid cultures in the 305 presence of cytokines FLT-3L, IL-7 and SCF and subjected to IP using PRMT1 Ab. The 306 immunoblot shows expression of PRMT1 using a PRMT1-Ab and HA-ETV6-RUNX1 using an HA-Ab in WCE and IP from wild-type FL cells. 